\title{
Effect of tamoxifen citrate on reproductive parameters of male dogs
}

\author{
Y. Corrada*, D. Arias, R. Rodríguez, \\ E. Spaini, F. Fava, C. Gobello \\ Small Animal Clinic \& Imaging Diagnose Service, Faculty of Veterinary Medicine, \\ National University of La Plata, La Plata, Argentina
}

Received 24 June 2003; accepted 31 July 2003

\begin{abstract}
Tamoxifen is a synthetic, nonsteroidal Type I antiestrogenic compound that competitively blocks estrogen receptors with a mixed antagonist-agonist effect. The manifestation of these different actions depends on each species, organ, tissue and cell type considered. Very little is known about the effect of antiestrogens in dogs. The objectives of this study were to determine the effects of tamoxifen citrate on some testis, prostate, hormone, and semen parameters in seven Beagle dogs with uncomplicated spontaneous benign prostatic hyperplasia. Two dogs were normospermic, four were oligozoospermic, and one was azoospermic. The dogs were allocated to a control pretreatment period, followed by a treatment period, and five post-treatment periods (the duration of each period was 4 weeks). During the treatment period, $2.5 \mathrm{mg}$ tamoxifen citrate was given p.o. daily for 28 days to all the dogs. Maximum scrotal width, testicular consistency, libido semen parameters, prostatic volume, serum testosterone concentrations, and side effects were assessed. Tamoxifen negatively affected testis size and libido $(P<0.01)$, and decreased prostatic volume $(P<0.02)$ and testosterone concentrations during treatment. Semen quality deteriorated to nadir values $(P<0.01)$ approximately one spermatic cycle after treatment and returned to pre-treatment values on the second cycle after treatment in all the dogs, except one young oligoazoospermic dog, in which the sperm count was higher $(P<0.01)$ at that time. No side effects were observed and fertility was conserved at the end of the study. Tamoxifen acted more like an agonist than antagonist on the gonadal axis and, therefore, upon both the prostate and testis. Therefore, tamoxifen may have therapeutic applications in dogs.
\end{abstract}

(C) 2003 Elsevier Inc. All rights reserved.

Keywords: Tamoxifen; Dog; Sperm; Prostate; Testis

\footnotetext{
${ }^{*}$ Corresponding author. Tel.: +54-221-425-2155; fax: +54-221-425-7980.

E-mail address: cgobello@fcv.unlp.edu.ar (Y. Corrada).
} 


\section{Introduction}

Tamoxifen is a synthetic nonsteroidal Type I antiestrogenic compound that competitively blocks estrogen receptors with a mixed antagonist-agonist effect. Type I estrogen antagonists partially inhibit the action of agonists, but due to their own agonistic properties, they also induce some estrogenic responses [1]. The manifestation of these different actions depends on each species, organ, tissue, and cell type [1]. In humans, tamoxifen exerts antiestrogenic activity on the mammary gland and agonistic effects on the uterus [2]. The exact mechanisms of this duality are not completely understood, but they may depend on the expression of specific cell estrogen receptor variants [1]. Adverse reactions of antiestrogens described in humans include nausea, vomiting, vertigo, and weight gain [3]. Very little is known about the effect of antiestrogens in dogs, and all the studies have been carried out in bitches, where it acts more like an agonist than antagonist [4-6].

Antiestrogens, e.g. clomiphen and tamoxifen, are prescribed for the treatment of idiopathic oligo/azoospermia in men [7]. In humans, antiestrogens interfere with the normal negative feedback of sex steroids at the hypothalamus and pituitary, thereby increasing endogenous gonadotropin-releasing hormone $(\mathrm{GnRH})$ secretion from the hypothalamus and follicle stimulating hormone (FSH) and luteinizing hormone (LH) secretion directly from the pituitary. In turn, LH stimulates Leydig cells in the testes; this has been claimed to increase local testosterone $\left(\mathrm{T}_{2}\right)$ production, thereby boosting spermatogenesis, with a possible improvement in fertility $[8,9]$.

There are contradictory data in the literature regarding improvement of semen quality with these treatments in humans [10-13]; similar studies in dogs are lacking. It has been hypothesized that dogs with partial seminiferous tubule failure in which FSH is still normal or slightly elevated may respond to clomiphene or tamoxifen treatment and drug regimes have been recommended for such dogs [14].

Tamoxifen citrate has also been used empirically by practitioners for the treatment of benign prostatic hyperplasia (BPH) in dogs (unpublished data), although no controlled trial has been carried out to test its efficacy and safety for this purpose. Development of safe and economic alternatives to medically manage canine BPH are necessary for dogs in which orchidectomy is risky or undesirable.

The objective of this study was to test the effect of tamoxifen citrate upon some testis, prostate, hormone, and semen parameters in dogs.

\section{Materials and methods}

\subsection{Animals}

Seven clinically healthy Beagle dogs, 4-11 years of age, weighing 12-14 kg, were used for a 28-week interval. The dogs had been living in kennels (with outside access), fed a commercial dog food and given water ad libitum, and were previously trained for manual semen collection.

All dogs had normal libido and testosterone response after hCG challenge [15] and presented spontaneous uncomplicated BPH (prostatic volume $>10 \mathrm{ml}$ [16] and negative 
sperm routine culture). Two dogs were normospermic (total sperm count $>200 \times 10^{6}[15]$ ), four were oligozoospermic (total sperm count $<200 \times 10^{6}[15,17]$ ) and one was azoospermic.

\subsection{Pre-treatment, treatment, and post-treatment periods}

The dogs were used as their own controls and, therefore, allocated to a control pre-treatment period, followed by a treatment period and five post-treatment periods of 4 weeks each; therefore, there were approximately two sperm cycles of follow up after the treatment period [18]. During the treatment period, tamoxifen citrate (Tamoxifeno ${ }^{\mathbb{R}}$, Filaxis, Martinez, Argentina) was given at a total dose of $2.5 \mathrm{mg}$ p.o. daily for 28 days to all dogs.

\subsection{Testicular and libido examination}

Maximum scrotal width $(\mathrm{cm})$, testicular consistency, and libido were evaluated once weekly throughout the study. Testicular consistency was classified as normal, soft, or firm. Libido (sexual arousal during manual stimulation) was subjectively assessed on a scale of $0-3$, where 3 represented rapid erection and thrusting movements, 0 represented neither erection nor ejaculation, and 1 and 2 were gradations between.

\subsection{Semen analysis}

Semen was collected weekly throughout the study by manual stimulation in the presence of a bitch. The second and third fractions were collected in prewarmed glass tubes and volume recorded. A drop of second fraction was placed on a warmed glass slide and the percentage of spermatozoa with forward progressive motility was assessed subjectively at $400 \times[19]$ and classified according to their vigor as normal progressive (3), slow motion (2), moving locally (1), or nonmotile (0).

Total sperm number was assessed in an aliquot of semen diluted 1:100 using an improved Neubauer hemacytometer counting chamber and the total sperm output was calculated by multiplying the concentration by the volume of the second fraction.

Morphology was evaluated by smearing a drop of semen on a glass slide, allowing the slide to dry, and staining with Giemsa stain and examining under magnification more than 100 spermatozoa [19-21]. Percentage of morphologically normal sperm and sperm with primary and secondary abnormalities were determined in each sample [19].

\subsection{Transabdominal prostatic ultrasonography}

Prostatic volume $(\mathrm{ml})$ was assessed on the last week pre-treatment and treatment periods, and then on Weeks 5, 10, 14, and 19 after treatment by transabdominal ultrasonography (Pie Medical S100, $5 \mathrm{MHz}$ transducer, Maastricht, The Netherlands) according to Kamolpatana et al. [22]. Ultrasonographic character of prostatic parenchyma was also monitored and classified as normal or hyperechoic [23]. 


\subsection{Blood sampling and hormone assay}

Blood samples from peripheral venepuncture were drawn before and $4 \mathrm{~h}$ after $500 \mathrm{IU}$ i.m. of human chorionic gonadotropic (hCG; Endocorion ${ }^{\circledR}$, Elea, BsAs, Argentina) for $\mathrm{T}_{2}$ determinations [15] before and on Week 4 of treatment and 28 days after treatment in four of the dogs. All the samples were centrifuged for $15 \mathrm{~min}$, serum obtained and stored at $20{ }^{\circ} \mathrm{C}$ until assayed.

Serum $\mathrm{T}_{2}$ was measured by radioimmunoassay, using a solid phase kit (Coat-A-Count, $\mathrm{DPC}^{\circledR}$, Los Angeles, CA, USA). For this kit, the sensitivity at $95 \%$ binding was $0.04 \mathrm{ng} / \mathrm{dl}$ and the intra-assay $\mathrm{CV}$ was $5.9 \%$. All samples were measured in the same assay.

\subsection{Post-treatment fertility}

Post-treatment fertility was assessed in three dogs by permitting two natural matings every $72 \mathrm{~h}$ to previously fertile female Beagles during behavioral estrus 17-20 weeks after treatment.

\subsection{Side effects}

The presence of previously reported digestive side effects was recorded during the 28 weeks of the study in all the dogs [3]. Blood samples were taken as described above and collected in tubes with EDTA as anticoagulant for complete blood count analysis and platelet count once weekly for 6 weeks beginning 1 week before tamoxifen treatment in four dogs. The analyses were performed on the day of collection.

\subsection{Statistical analysis}

No significant differences (PROC GLM, SAS ${ }^{\circledR} ; 1989$ ) in all the studied parameters, except for sperm count, were found among dogs at the beginning of the study and, therefore, they were analyzed together.

Prostatic volume percentage changes [((value of different week (treatment, and 5, 10, 14, and 19 after treatment) - value of pre-treatment week)/pre-treatment week) $\times 100$ ] were analyzed by least squares analysis of variance using the General Linear Model Procedure (PROC GLM, SAS $\left.{ }^{\circledR} ; 1989\right)$. The mathematical model included the main effect of week (pretreatment, treatment, and 5, 10, 14, and 19 after treatment, $n=6$ ).

Scrotal width, sperm fraction volumes, primary and secondary semen abnormalities, and motility during the pre-treatment, treated, and the five post-treatment periods (1 [Weeks 1-4]; 2 [Weeks 5-8]; 3 [Weeks 9-12]; 4 [Weeks 13-16]; and 5 [Weeks 17-20]) were analyzed by the same procedure as described before but including in the mathematical model the main effect of period (pre-, treated, and the five post-treatment periods, $n=7$ ). Sperm count was analyzed for each group (normal count $[n=2]$ and oligozoospermic $[n=4])$ by the same procedure. In all cases, orthogonal contrasts were used to test differences among selected biologically significant week or periods, respectively.

The frequency of dogs achieving side effects (present or absent), different grades of libido $(0,1,2,3)$ and sperm motility vigor $(0,1,2,3)$, testicular consistency (normal or 
abnormal) and post-treatment fertility in the different periods were analyzed in all the dogs $(n=7)$ by categorical data analysis (PROC CATMOD, SAS $\left.{ }^{\circledR} ; 1988\right)$.

Descriptive statistic for blood parameters was carried out by (PROC MEANS, SAS ${ }^{\circledR}$; 1988). In all cases, results were expressed in least squares means (LSM) \pm S.E.M. The level of significance was set at 0.05 .

\section{Results}

Prostatic ultrasonographic volume changed during the study period $(P<0.02)$; it quickly decreased in all dogs, so that it was significantly different from pre-treatment values. Then, prostatic volume increased gradually and came back to pre-treatment values 5 weeks after treatment (Fig. 1). Prostatic parenchyma appeared normal to uniformly hyperechoic throughout the study.

Volume of the third (prostatic) fraction of the ejaculate decreased $(P<0.01)$ to a few drops or was absent during and 1 month after treatment. It subsequently returned gradually to pre-treatment values and was higher $(P<0.01)$ at the end of the study (Fig. 2). Maximum scrotal width began to significantly decrease during the treatment period and was the least on the first month after treatment, then it increased to pre-treatment values $(P<0.01)$. Testes became soft $1-2$ weeks after the beginning of tamoxifen treatment to 4

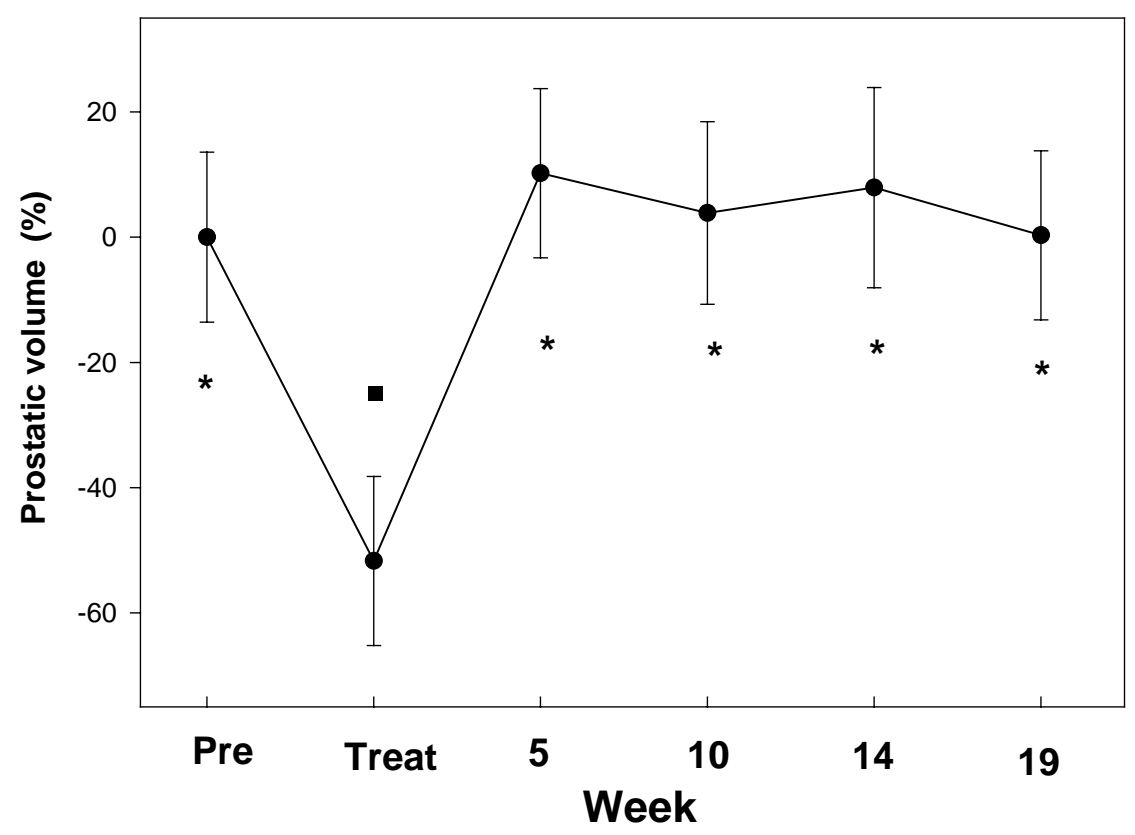

Fig. 1. Prostatic volume percentage change (LSM \pm S.E.M.) assessed by ultrasonography in seven Beagles dogs treated with tamoxifen citrate for 28 days. Days were monitored 4 weeks before treatment and 19 weeks after treatment. Different symbols indicate biologically selected statistically significant differences. 


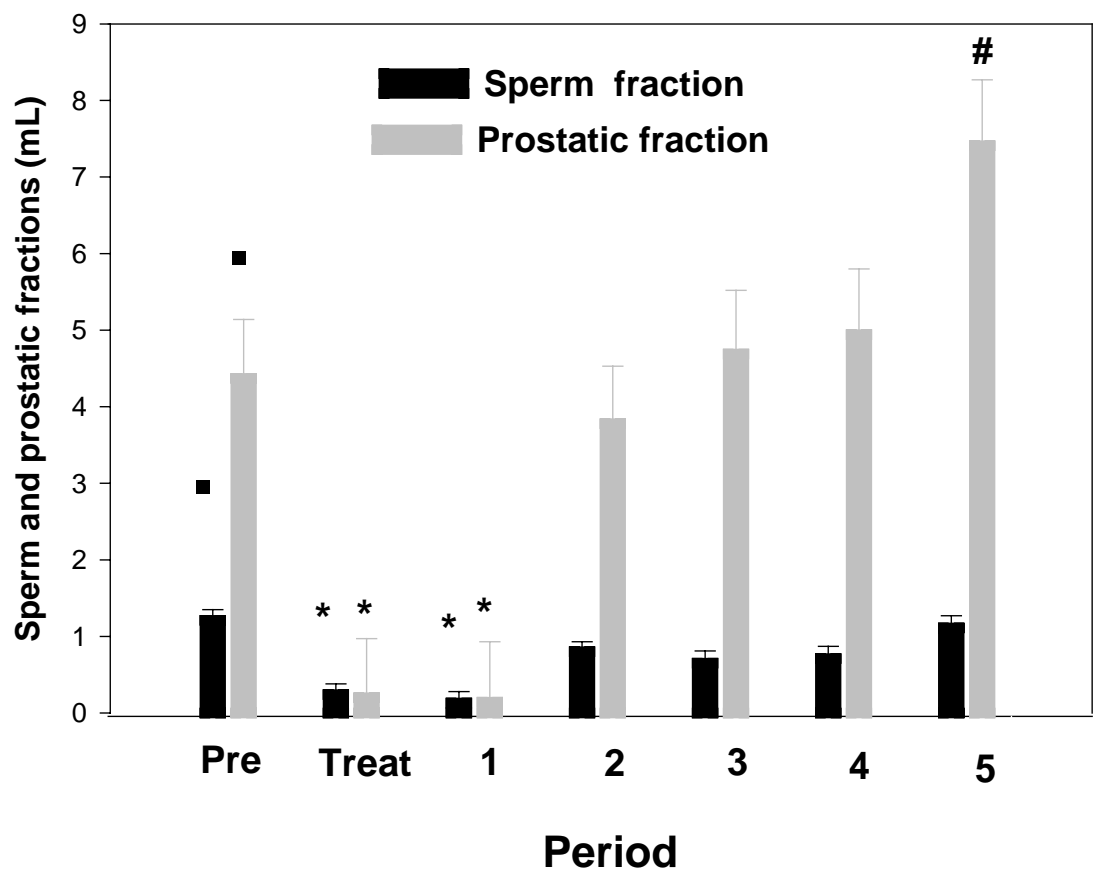

Fig. 2. Second and third fractions of the ejaculate (LSM \pm S.E.M.) of the dogs of Fig. 1, followed for five 1month periods, are shown as black and grey columns, respectively. Different symbols indicate biologically selected statistically significant differences.

weeks after the end of the treatment; consistency then returned to pre-treatment normalcy (Fig. 3).

Libido decreased $(P<0.01)$ from the second week after beginning of the treatment, was nonexistent in most dogs at the end of treatment, and then returned gradually to pre-treatment behavior from Week 2 after treatment (Fig. 4).

Volume of the second (spermatic) fraction of the ejaculate began to decrease from the second week of treatment and was nearly absent during and the first period after treatment, and then increased to pre-treatment volumes $(P<0.01$; Fig. 2$)$. In some dogs, the second and third fractions of the ejaculate were difficult to distinguish and separate during the first ejaculations after treatment-induced aspermia.

Sperm count in the two normal dogs was significantly different among the different periods $(P<0.01$; Fig. 5). The pre-treatment period was significantly higher than treatment and the first two periods after treatment, and then increased gradually to pre-treatment values by the end of the study.

The azoospermic dog remained the same throughout the study. In the four oligoazoospermic males a different response was clearly seen between the three old dogs ( $>7$ years) and the remaining young dog (4 years). They were, therefore, illustrated and analyzed separately. In both subgroups (young and old) sperm count changed $(P<0.01)$ as in normal dogs, up to the fourth period after treatment. However, in the last period of the 


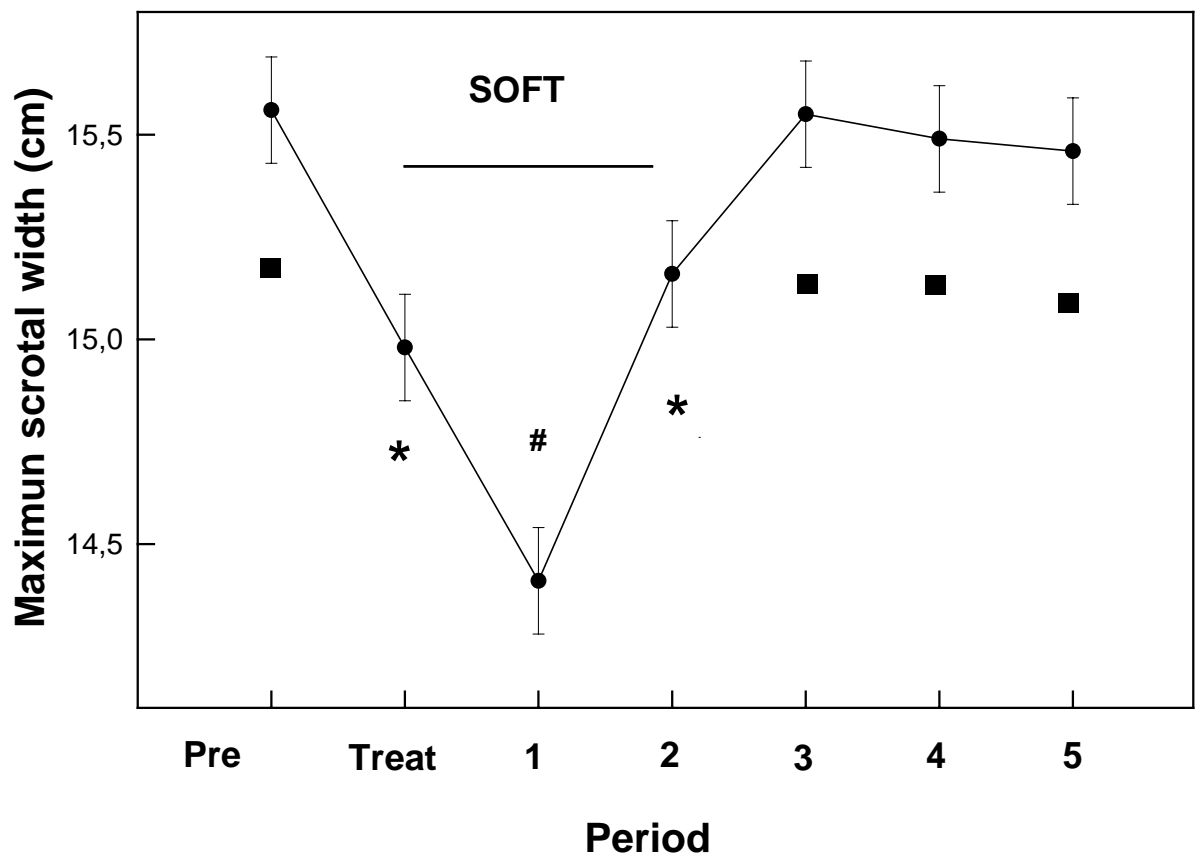

Fig. 3. Maximum scrotal width (LSM \pm S.E.M.) of the dogs of Figs. 1 and 2. The segment indicates the interval when the testes had a soft consistency. Different symbols indicate biologically selected statistically significant differences.

study, in the young dog sperm count increased $(P<0.01)$ compared to pre-treatment values, whereas in the old dogs it returned to the pre-treatment level (Fig. 6).

Significant reductions in sperm motility and vigor were present in all dogs up to two periods after treatment, when they reached the nadir values. Then, they returned gradually to pre-treatment values (or even higher) at the end of the study (Fig. 7).

The percentage of primary morphological abnormalities significantly increased from the treatment period up to two periods after treatment, when they reached a maximum, with a subsequent decreased (not significant) to values slightly below pretreatment at the end of the study. The change was partly due to a marked increase in the number of spermatozoa with head abnormalities and proximal cytoplasmic droplets. Secondary abnormalities did not change significantly during the study, although they increased rapidly during treatment, and then regained pre-treatment percentages (Fig. 8). Among the secondary abnormalities tail defects (bent and coiled tails) predominated.

Serum (LSM \pm S.E.M.) $\mathrm{T}_{2}$ concentrations before and after hCG challenging tests before, during, and one period after treatment are shown in Fig. 9.

Tamoxifen citrate was well tolerated by all the dogs and no clinical side effects were observed during the study. All hemogram and platelet parameters remained within the normal range during the study period. The bitches that were bred with the three dogs that gained normal sperm count (two normospermic and the young oligospermic) between 17 and 20 weeks after treatment became pregnant and whelped normal litters. 


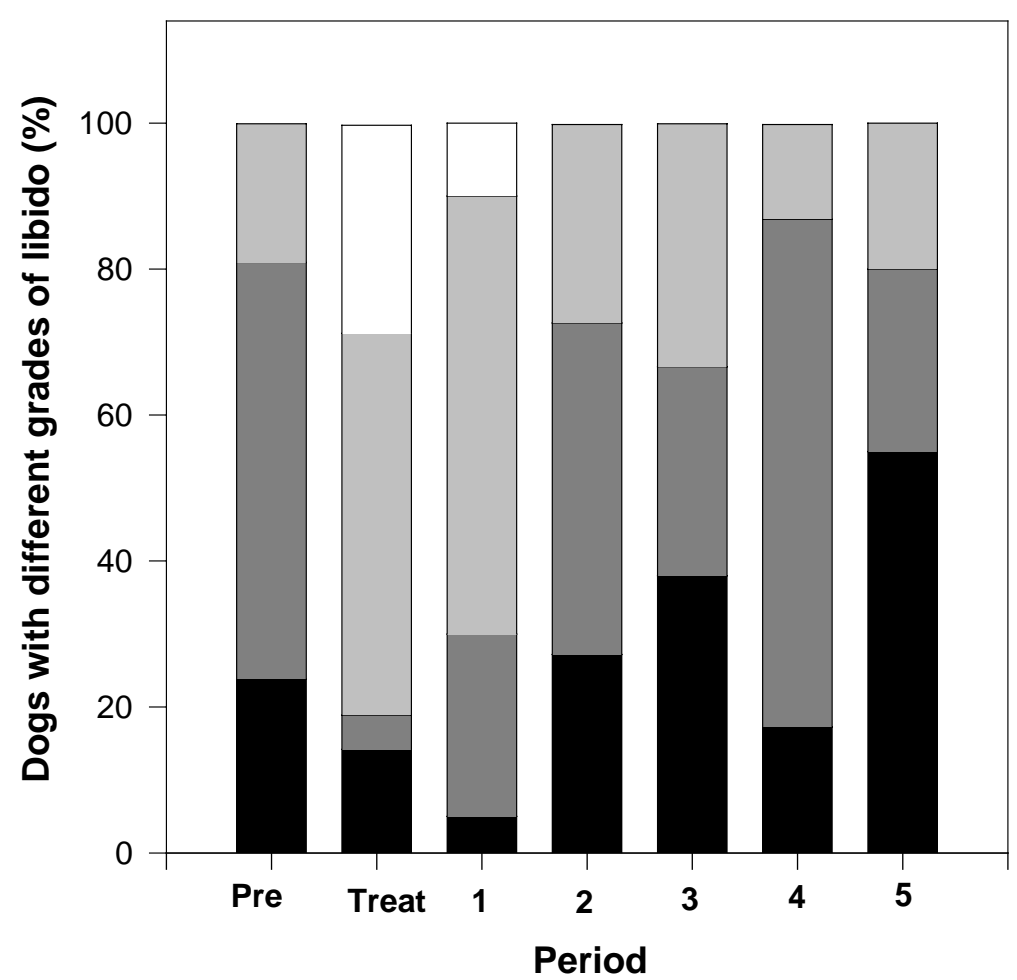

Fig. 4. Libido of the dogs of Figs. 1 and 2. In each column, the total number of dogs is represented as the $100 \%$. At each period (pre-treatment, treatment, and the five post-treatment periods), the proportion of dogs with a specific grade of libido is represented as follows: black, dark grey, light grey, and white for grades $3,2,1$, and 0 , respectively.

\section{Discussion}

BPH is a common, age-related condition in dogs, with $80 \%$ of intact males over 5 years affected [24]. Although castration is an effective treatment for BPH, economic, effective, fast-acting therapeutic protocols are still needed for dogs at high risk for surgery or for those that may be subsequently used for breeding. Several medical options, like progestagens, estrogens, and $5 \alpha$-reductase inhibitors, have been indicated for BPH [25]. Longterm progestagen treatment effects on pancreatic and adrenocortical function are unknown. Estrogens may cause prostatic enlargement due to squamous cell metaplasia and also suppress hematopoesis [26]. Finasteride, a $5 \alpha$-reductase inhibitor, is expensive and has a slow action, with a delay of at least 2 months before an effect is evident [16].

The effect of antiestrogens, like tamoxifen, on the hyperplasic prostate has not been previously described in dogs. In this study, tamoxifen rapidly decreased prostastic volume, resembling the action previously described for ethinyestradiol [26]. Like low doses of estrogens, tamoxifen could indirectly affect the prostate by inhibiting the gonadoptropic activity of the anterior pituitary gland through a negative feedback mechanism, thereby 


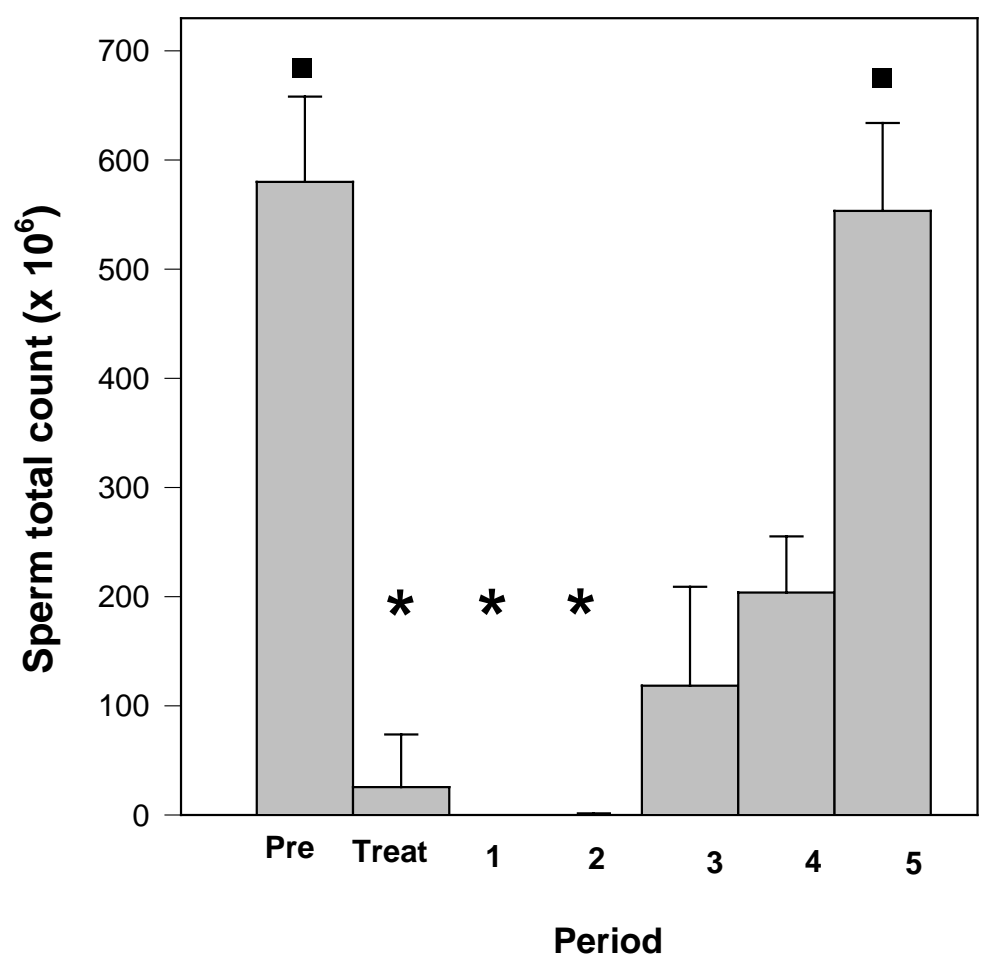

Fig. 5. Total sperm count (LSM \pm S.E.M.) of the two dogs with pre-treatment normal sperm of Figs. 1 and 2. Different symbols indicate biologically selected statistically significant differences.

decreasing androgen production, with a subsequent decrease in prostatic size [26]. The temporary suppression of prostatic secretion was due to reduced gland volume.

Although further work with prolonged administration on a larger number of animals is necessary, this tamoxifen therapeutic protocol could be considered a safe and effective therapeutic option when a quick response is necessary (e.g. obligate postponement of orchidectomy to improve the general health of a dog, acute prostatitis associated with $\mathrm{BPH})$. A rapid treatment response for this purpose has not been previously obtained with other drugs [16,26,27].

Oligospermia and azoospermia are possible findings of the breeding soundness examination or the pre-freezing semen evaluation of stud dogs. Although they can be caused by a wide variety of etiologies [28] often no reason can be identified and they are classified as idiopathic. In dogs with testicular problems in which specific therapy is not possible, a great variety of therapeutic measurements, including antiestrogens, have been suggested as an extrapolation from treatments in men [14].

The effect on germinal epithelium, duration and dose of tamoxifen in dogs has not been established. The dose of tamoxifen used in this trial $(\sim 0.2 \mathrm{mg} / \mathrm{kg})$ was selected according to previous pilot studies (unpublished data). The etiology of the azoospermia and 


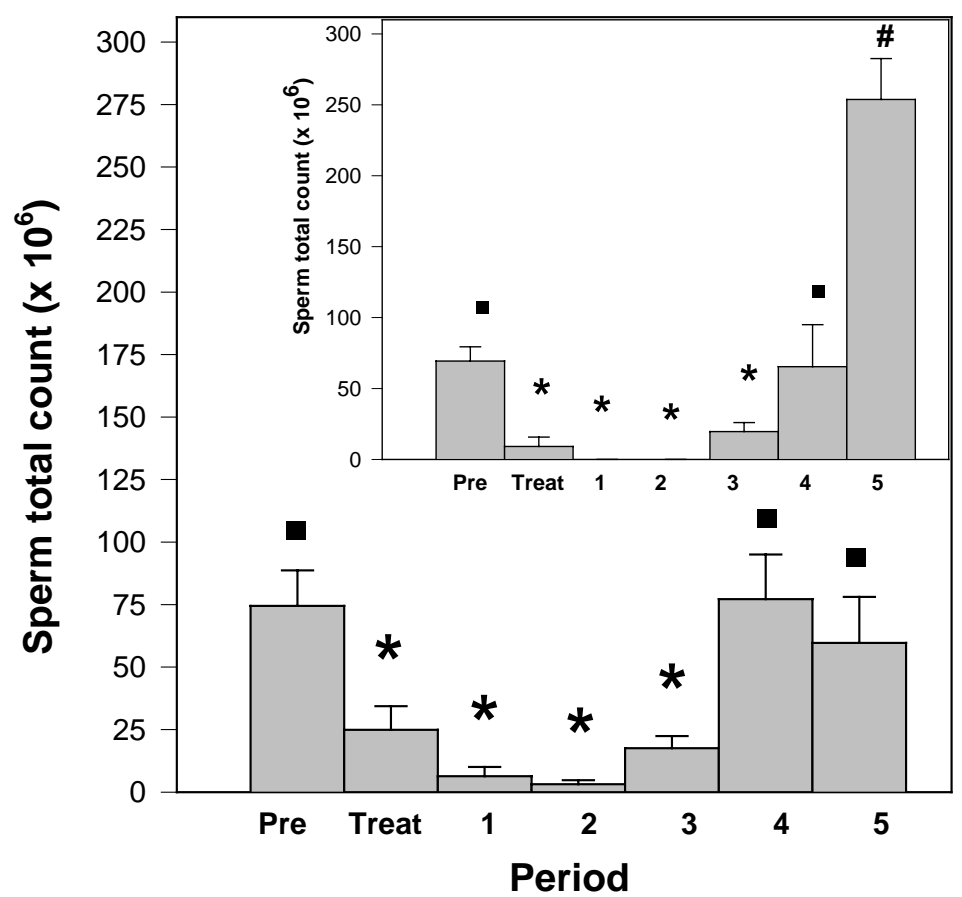

Fig. 6. Total sperm count (LSM \pm S.E.M.) of the three oligoazoospermic, old dogs of Figs. 1 and 2. Inset: total sperm count (LSM \pm S.E.M.) of the young oligoazoospermic dog of the same study. Different symbols in both panels indicate biologically selected statistically significant differences.

oligozoospermia in these otherwise healthy dogs was not established but could have been due to a physiological, age-related phenomenon in at least four of the dogs $[29,30]$.

Tamoxifen treatment led to softening and a reduction in size of testes during and up to 1 month after treatment. Soft testes have been associated with abnormal spermatogenesis [31]. Except in the azoospermic dog that remained the same throughout the study, sperm count in all the dogs was nil or decreased to far below that required for fertility [32] for approximately one spermatic cycle after treatment and subsequently returned to pre-treatment concentrations on the second spermatic cycle.

Semen deterioration and lack of libido was probably due to the suppression of gonadotrophin release, with a concomitant negative effect on spermatogenesis. These results resembled in time and response to those provoked by low doses of ethinylestradiol used for 4 weeks in three dogs in a previous study [26].

Based on the present results, with further testing, tamoxifen may become an effective, safe, and quick method of reversible contraception in this species. High doses of progestins or androgen administration have shown to suppress spermatogenesis in dogs while preserving libido [33]. The control of male sexual behavior is essential when treating working or show dogs and even male dogs housed with intact females. Moreover, the reversibility of this protocol was confirmed by proved fertility in all the dogs tested. 


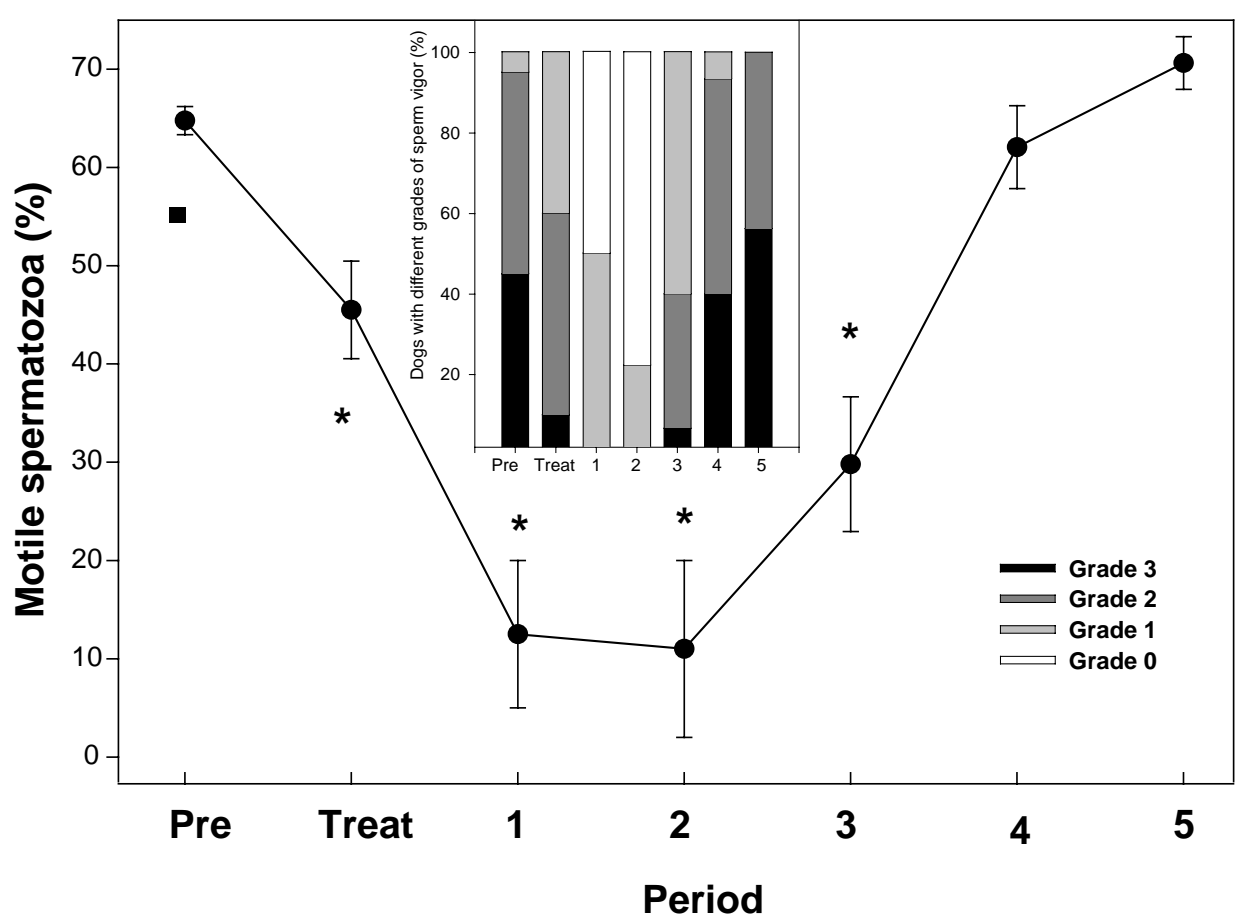

Fig. 7. Percentage of sperm motility (LSM \pm S.E.M.) of the dogs of Figs. 1 and 2. Different symbols indicate some of the statistically significant differences. Inset: sperm vigor of the same animals. In each column the total number of animals is represented as the $100 \%$. At each period the proportion of dogs with a given grade of libido is represented in different grey scales.

An increase in head defects and proximal droplets and a decrease in second fraction volume peaked one spermatic cycle after treatment. Therefore, tamoxifen affected testes probably by suppression of gonadotrophin release, with a concomitant negative effect on spermatogenesis.

That secondary abnormalities first appeared 7 days after initiation of treatment indicated that these defects arose during the epididymal transit and that tamoxifen, directly or indirectly affected epididymal function, similar to ethinylestradiol [26]. The decrease in sperm motility and vigor also indicated that epididymal function rapidly deteriorated during treatment. In most species, the time taken for spermatozoa to pass through epididymis is approximately 14 days [34].

The only one young oligoazoospermic dog used in this study had an increased to normal sperm count on the second spermatic cycle after treatment. Age-related differences on semen effects were previously reported in dogs treated with low doses of ethinylestradiol [26]. Testosterone has been used in oligospermic men to cause azoospermia and when stopped, caused an increase in total sperm output higher than at the beginning of the therapy [35]. Furthermore, in all dogs, a slight improvement in motility, vigor, and primary abnormalities were detected at the end of the study. These results warrant further studies in a larger number of oligoazoospermic animals to 


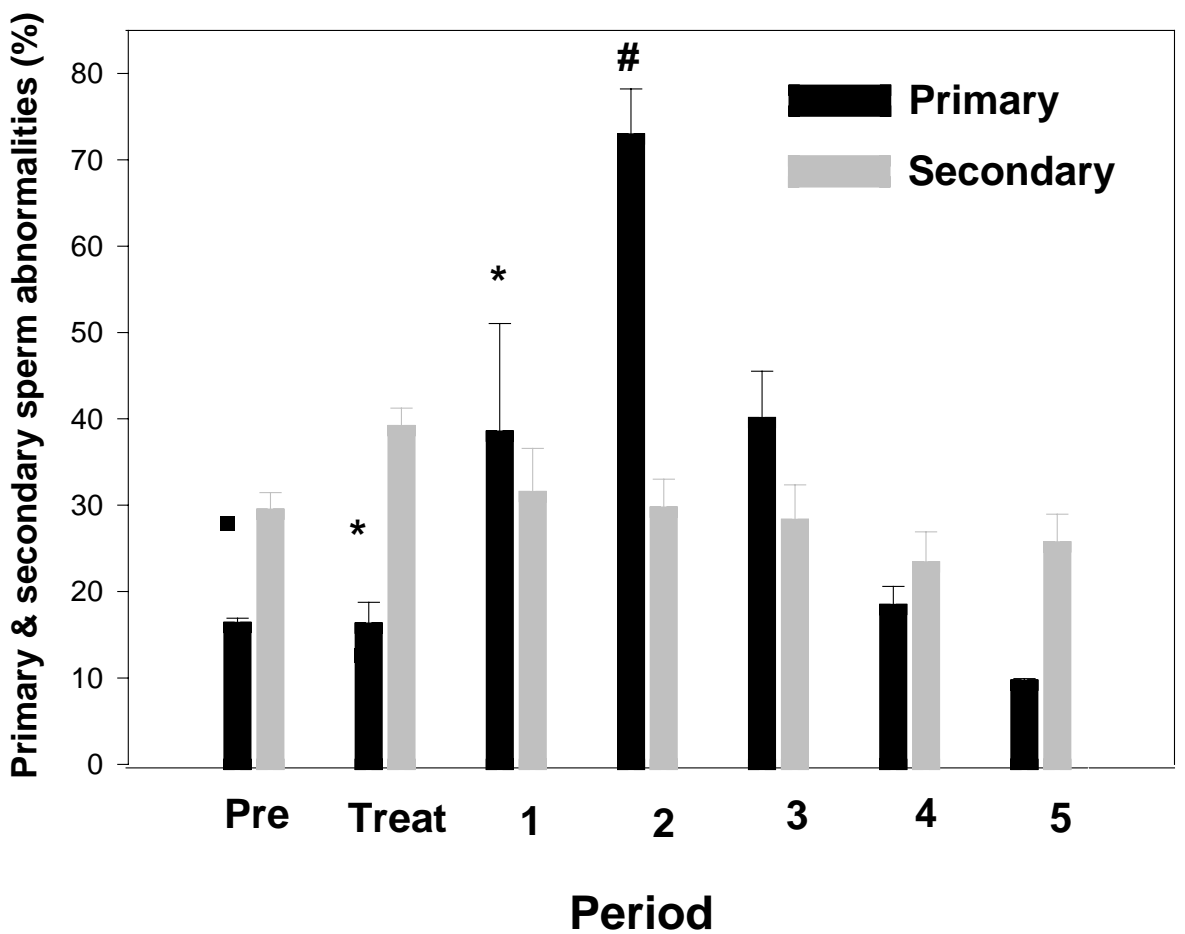

Fig. 8. Percentage of primary and secondary sperm abnormalities (LSM \pm S.E.M.) of the dogs of Figs. 1 and 2. Different symbols indicate biologically selected statistically significant differences.

test the efficacy of tamoxifen to improve semen quality. The improvement of semen characteristics would not only be useful to optimize reproductive performance during breeding management, but also when freezing semen or using any assisted reproductive technology.

Testosterone challenge tests were performed during different periods of the study to evaluate the hypothalamic-pituitary gonadal axes. Human chorionic gonadotrophin stimulation test permitted assessment of maximal testicular capacity to secrete $T_{2}$ [36]. In the pre-treatment period, post-hCG stimulation $\mathrm{T}_{2}$ had a normal increase $(>50 \% ;[15])$. A lack of normal response to hCG during treatment was compatible with an estrogen-like effect of tamoxifen on the gonadal axis [36]. A transient suppression of testicular synthesis of $\mathrm{T}_{2}$ may be considered during treatment, resulting in a slight stimulation of $\mathrm{T}_{2}$ synthesis in the post-treatment period. It is then hypothesized that tamoxifen may have an estrogenic effect in dogs, having a negative feedback effect at the hypothalamic-pituitary level, resulting in lower plasma FSH and LH levels and consequently lower plasma $\mathrm{T}_{2}$ concentrations. Conversely, when the tamoxifen effect is withdrawn, a temporary overshoot of the pituitary effects occurs.

Finally, it was important to determine whether this protocol caused estrogen-like suppression of the bone marrow. Consistent with previous studies with the same drug, no hematological abnormalities were found during the testing period $[6,26]$. 


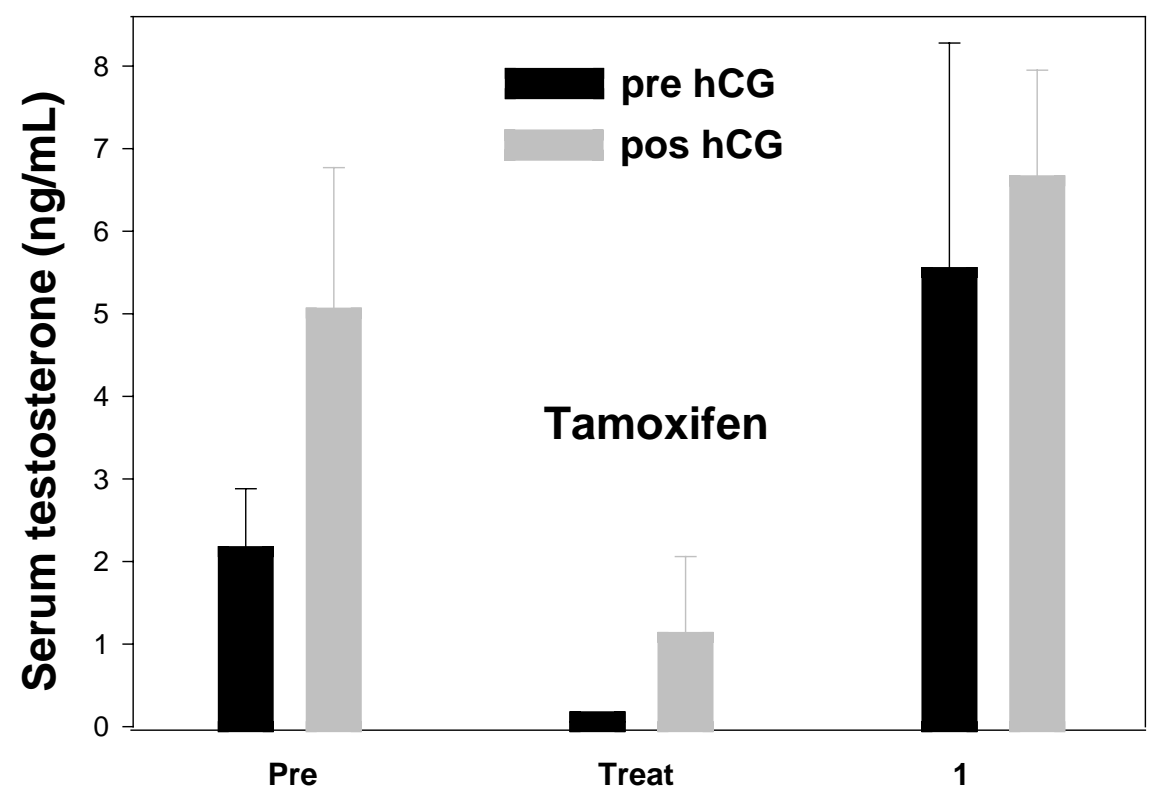

\section{Period}

Fig. 9. Serum testosterone concentrations (LSM \pm S.E.M.) before and $4 \mathrm{~h}$ after hCG (500 IU i.m.) challenge tests before, during, and one period after treatment in four of the dogs of Figs. 1 and 2.

In the present study, tamoxifen induced an estrogenic-like response, acting more like an agonist than antagonist on the gonadal axis and, therefore, upon both the prostate gland and testis. These effects, along with minimal adverse reactions, indicated that tamoxifen may have some therapeutic applications in this species that warrant further research.

\section{Acknowledgements}

The authors thank Filaxis, Argentina, for supplying tamoxifen citrate and Holiday Scott SA, Argentina, for providing dog food.

\section{References}

[1] Hoffmann B, Schuler G. Receptors blockers-general aspects with respect to their use in domestic animal reproduction. Anim Reprod Sci 2000;60/61:295-312.

[2] Jordan VC. The role of tamoxifen in the treatment and prevention of breast cancer. Curr Probl Cancer 1992;16:129-76.

[3] Willams CL, Stancel GM. Estrógenos y progestágenos. In: Herdman JJ, Limbird LE, Goodman, Gilman A, editors. Goodman \& Gilman's. Bases farmacológicas de la terapéutica. 9th ed. México: McGraw-Hill Companies Inc. Interamericana; 1996. p. 1489-529. 
[4] Gobello C, del Amo AN. Estrus induction with clomiphene citrate in bitches. In: First International Congress FCV-UNLP and VII International Veterinary Meeting, 1993 Nov 4-6; La Plata, Argentina.

[5] Morris JS, Dobson JM, Bostock DE. Use of tamoxifen in the control of canine mammary neoplasia. Gynecol Oncol 1992;39:82-4.

[6] Bowen RA, Olson PN, Young S, Withhrow SJ. Efficacy and toxicity of tamoxifen for prevention and termination of pregnancy in bitches. Am J Vet Res 1988;49:27-31.

[7] Sorbie PJ, Perez Marrero R. The use of clomiphene citrate in male infertility. J Urol 1984;131:425-9.

[8] Kato J, Kobuyashi T, Villee LM. Effect of clomiphene in the uptake of estradiol by the active hypothalamus and hypophysis. Endocrinology 1968;82:1049.

[9] Roy S, Mahesh VB, Greenblatt RE. Effect of clomiphene on the physiology of the reproduction in the rat. Acta Endocrinol (kbh) 1964;47:669.

[10] Check JH, Racoff AE. Improved fertility in oligospermic males treated with clomiphene citrate. Fertil Steril 1977;48:113-7.

[11] Epstein JA. Clomiphene treatment in oligospermic infertile males. Fertil Steril 1977;28:741-5.

[12] Ain Melk Y, Belisle S, Carmel M, Tetreault JP. Tamoxifen citrate in male infertility. Fertil Steril 1987;48:113-7.

[13] Newton R, Schinfeld JS, Schiff I. Clomiphene treatment of infertile men: failure of with idiopathic oligospermia. Fertil Steril 1980;34:399-400.

[14] Feldman EC, Nelson RW. Canine and feline endocrinology and reproduction. Philadelphia: WB Saunders; 1987. p. 548.

[15] Johnston SD, Root Kustritz MV, Olson PN. Clinical approach to infertility in the male dog. In: Canine and feline theriogenology. Philadelphia: WB Saunders; 2001. p. 370-87.

[16] Iguer Ouada M, Verstegen JP. Effect of finasteride on seminal composition, prostate function and fertility in male dogs. J Reprod Fertil 1997;51:139-49.

[17] Dahlbom M, Andersson G, Huszenicza G, Alanko M. Poor semen quality in Irish wolfhounds and spermatological study. J Small Anim Practice 1995;36:547-50.

[18] Foote RH, Swierstra EE, Hunt WC. Spermatogenesis in the dog. Anat Rec 1972;172:341-51.

[19] Johnston SD. Performing a complete canine semen evaluation in a small animal hospital. Vet Clin North Am (Small Anim Pract) 1991;21:545-51.

[20] Watson PF. Use of Giemsa stain to detect changes in acrosomes of frozen ram spermatozoa. Vet Rec 1975;97:12-3.

[21] Root MV, Johnston SD. Basis for a complete reproductive examination of the male dog. Vet Med Surg 1994;9:41-5.

[22] Kamolpatana K, Johnston RG, Johnston SD. Determination of canine prostatic volume using transabdominal ultrasonography. Vet Radiol Ultrasound 2000;41(1):73-7.

[23] Finn ST, Wrigley RH. Ultrasonography and ultrasound biopsy of the canine prostate. In: Kirk RW, editor. Current veterinary therapy. X. Philadelphia: WB Saunders; 1989. p. 1227-39.

[24] Johnston SD, Kamolpatana K, Root-Kustritz MV, Johnston GR. Prostatic disorders in the dog. Anim Reprod Sci 2000;60-61:405-15.

[25] Gobello C, Corrada Y. Non infectious disorders of canine prostatic diseases: a medical approach. Compend Contin Educ Pract Vet 2001;24(2):99-107.

[26] Bamberg-Thalen B, Linde-Forsberg C. The effects of medroxyprogesterone acetate and ethinylestradiol on hemogram, prostate, testes and semen quality in normal dogs. J Vet Med A 1992;39:264-70.

[27] Sirinarumitr K, Johnston SD, Root-Kustritz MV, Johnston GR, Sarkar DK, Memom MA. Effects of finasteride on size of the prostate gland and semen quality in dogs with benign prostatic hypertrophy. J Am Vet Assoc 2001;218:1275-80.

[28] Olson PN. Clinical approach for evaluating dogs with azoospermia or aspermia. Vet Clin North Am (Small Anim Pract) 1991;3:591-608.

[29] Dahlbom M, Andersson G, Juga J, Alanko M. Fertility parameters in male Irish wolfhounds: a two year follow up study. J Small Anim Practice 1997;38:547-52.

[30] Soderberg SF. Infertility in the male dog. In: Current therapy in theriogenology. Philadelphia: WB Saunders; 1986. p. 544-8.

[31] Olson PN, Schultheiss P, Seim H. Clinical and laboratory finding associated with actual or suspected azoospermia in dogs: 18 cases. JAVMA 1992;20:478-82. 
[32] Oettle EE. Sperm morphology and fertility in the dog. J Reprod Fertil 1993;47:257-60.

[33] England GC. Effects of progesterone and androgens upon spermatogenesis and steroideogenesis in dogs. J Reprod Fertil 1997;51:123-38.

[34] Austin CR, Short RV. Germ cells and fertilization. In: Reproduction in mammals. London: Cambridge University press; 1972. p. 82.

[35] Ganong WF. The gonads: development and function of the reproductive system. In: Ganong W, editor. Review of medical physiology.10th ed. Los Altos, CA: Lange Medical Publications; 1981. p. 331-63.

[36] Shille VM, Olson PN. Dynamic testing in reproductive endocrinology. In: Kirk RW, Bonagura JD, editors. Current veterinary therapy. X. Philadelphia: WB Saunders; 1989. p. 1282-8. 